\title{
Role of Regular Exercise in Preventing Recurrent Malaria Attacks in Male and Female
}

\section{Seriki $\mathrm{SA}^{1 *}$, Adebayo $\mathrm{OF}^{2}$ and Enne $\mathrm{EV}^{2}$}

${ }^{1}$ Department of Human Physiology, College of Medical Sciences, Edo University, Nigeria

${ }^{2}$ Department of Human Physiology, College of Medicine, Bingham University, Nigeria

*Corresponding author: Seriki Samuel Adinoyi, Department of Human Physiology, College of Medical Sciences, Edo University, Iyamho, Nigeria, Email: seriki.samuel@edouniversity.edu.ng

\section{Research Article \\ Volume 3 Issue 1}

Received Date: March 06, 2020

Published Date: May 28, 2020

DOI: $10.23880 /$ aabsc- 16000147

\section{Abstract}

The entire human body is generally affected by exercise. Exercise affects the physiology of every system leading to various changes which generally affect the health of the body. Malaria on the other hand is one of the diseases with the most mortality rates especially in Nigeria and Africa, killing millions every year. As lethal as malaria is, no vaccine has been produced for it. Other preventive measures that improve individual's natural immunity against attacks are still the most effective ways of tackling the disease in the face of failing Artemisinin Combination Therapy (ACT) approved by the World Health Organisation (WHO) to manage patients attacked by the disease. This study examines the role of regular exercise on malaria occurrence among selected male and females. It was to determine whether or not regular exercise reduces the frequency of malaria occurrence? Sixty (60) healthy subjects were selected and divided into two equal groups of regularly exercising subjects and nonexercising subjects with each group consisting of an equal number of male and female subjects. The study was for a period of twelve (12) weeks with the subjects checked every two weeks to see if there was an incidence of malaria. The subjects then filled a questionnaire to ascertain other factors like duration of exercise. At the end of the study period, the non-exercising group showed a higher frequency of malaria attack in both the male and female subjects compared to their regularly exercising counterparts; the non-exercising female subjects had a mean frequency of malaria occurrence of 1.2 compared to 0.4 for the regularly exercising subjects, while the non-exercising male subjects had a mean frequency of malaria occurrence of 0.7 compared to 0.3 of the regularly exercising male subjects. The study showed that regular exercise reduces the frequency of malaria occurrence in individuals.

Keywords: Malaria Attack; Regular Exercise; Immunity; Sedentary Lifestyle

\section{Introduction}

Exercise is a physical activity that carries a lot physiological benefits in the human body. Exercise may also be directed to improvement of a person's general health, physical fitness, or as physical therapy, to augment an existing treatment to remedy or to ameliorate the effects of a disease or illness upon the body. Virtually every process and organ within the body is affected by exercise. For instance, the skin, the largest human organ, undergoes physical changes when exposed to the environmental factors encountered in sport, such as increases and decreases in external temperatures.

The results of reviewed studies on the health benefits of exercise indicate that physical activity seems to be an important factor that can have beneficial effects on noncommunicable disease such as weight gain and obesity, coronary heart diseases, Type II diabetes mellitus and the age-related impairments like dementia and Alzheimer's disease. Exercise has also been shown to have positive effects on mood, depression and mental activity. 


\section{Types of Exercise}

Exercises are generally grouped into two types depending on the overall effect they have on the human body:

Aerobic exercise, such as cycling, walking, running, hiking, and playing tennis, focus on increasing cardiovascular endurance [1].

Anaerobic exercise, such as weight training, increase short-term muscle strength. Aerobic exercise has the most valuable effects on the general health of the body.

Malaria: Malaria is a non-communicable disease transmitted by the female Anopheles mosquito caused by parasitic protozoans (a group of single-celled microorganisms) belonging to the Plasmodium type (WHO). The term malaria originates from Medieval Italian: mala aria-"bad air"; the disease was formerly called ague or marsh fever due to its association with swamps and marshland. The term first appeared in the English literature about 1829. Malaria was once common in most of Europe and North America, where it is no longer endemic, though imported cases do occur [2].

Scientific studies on malaria made their first significant advance in 1880, when Charles Louis Alphonse Laveran a French army doctor working in the military hospital of Constantine in Algeria observed parasites inside the red blood cells of infected people for the first time. He therefore proposed that malaria is caused by this organism, the first time a protist was identified as causing disease [3].

For this and later discoveries, he was awarded the 1907 Nobel Prize for Physiology or Medicine. A year later, Carlos Finlay, a Cuban doctor treating people with yellow fever in Havana, provided strong evidence that mosquitoes were transmitting disease to and from humans. This work followed earlier suggestions by Josiah C. Nott, and work by Sir Patrick Manson, the "father of tropical medicine", on the transmission of filariasis [3].

\section{Symptoms of Malaria}

Malaria causes symptoms that typically include fever, fatigue, vomiting, and headaches. In severe cases it can cause yellow skin, seizures, coma, or death. Symptoms usually begin ten to fifteen days after being bitten. If not properly treated, patient may have recurrences of the disease months later. In those who have recently survived an infection, reinfection usually causes milder symptoms. This partial resistance disappears over months to years if the person has no continuing exposure to malaria [4].

The current study is to examine how regular exercise affects occurrence of malaria, by determining how often regularly exercising subjects go down with malaria compared to non-exercising subjects, and to find out the effect of regular exercise on the occurrence of malaria.

\section{Complications}

Malaria has several serious complications. Among these is the development of respiratory distress, which occurs in up to $25 \%$ of adults and $40 \%$ of children with severe P. falciparum malaria. Possible causes include respiratory compensation of metabolic acidosis, non-cardiogenic pulmonary oedema, concomitant pneumonia, and severe anaemia. Although rare in young children with severe malaria, acute respiratory distress syndrome occurs in 5-25\% of adults and up to $29 \%$ of pregnant women [5]. Co-infection of HIV with malaria increases mortality. Renal failure is a feature of black water fever, where hemoglobin from lysed red blood cells leaks into the urine [6].

Infection with P.falciparum may result in cerebral malaria, a form of severe malaria that involves encephalopathy. It is associated with retinal whitening, which may be a useful clinical sign in distinguishing malaria from other causes of fever [7]. Enlarged spleen, enlarged liver or both of these, severe headache, low blood sugar, and hemoglobin in the urine with renal failure may occur. Complications may include spontaneous bleeding, coagulopathy, and shock [7].

Malaria in pregnant women is an important cause of stillbirths, infant mortality, abortion and low birth weight, particularly in P. falciparum infection, but also with $P$. vivax [7].

\section{Life Cycle of Malaria Parasite}

A mosquito causes an infection by a bite. First, sporozoites enter the bloodstream, and migrate to the liver. They infect liver cells, where they multiply into merozoites, rupture the liver cells, and return to the bloodstream. The merozoites infect red blood cells, where they develop into ring forms, trophozoites and schizonts that in turn produce further merozoites. Sexual forms are also produced, which, if taken up by a mosquito, will infect the insect and continue the life cycle.

In the life cycle of Plasmodium, a female Anopheles mosquito (the definitive host) transmits a motile infective form (called the sporozoite) to a vertebrate host such as a human (the secondary host), thus acting as a transmission vector. A sporozoite travels through the blood vessels to liver cells (hepatocytes), where it reproduces asexually (tissue schizogony), producing thousands of merozoites. These infect new red blood cells and initiate a series of asexual multiplication cycles (blood schizogony) that produce 8 to 
24 new infective merozoites, at which point the cells burst and the infective cycle begi. Other merozoites develop into immature gametocytes, which are the precursors of male and female gametes. When a fertilized mosquito bites an infected person, gametocytes are taken up with the blood and mature in the mosquito gut. The male and female gametocytes fuse and form an ookinete; a fertilized, motile zygote. Ookinetes develop into new sporozoites that migrate to the insect's salivary glands, ready to infect a new vertebrate host. The sporozoites are injected into the skin, in the saliva, when the mosquito takes a subsequent blood meal [8].

Only female mosquitoes feed on blood; male mosquitoes feed on plant nectar, and do not transmit the disease. The females of the Anopheles genus of mosquito prefer to feed at night. They usually start searching for a meal at dusk, and will continue throughout the night until taking a meal [9]. Malaria parasites can also be transmitted by blood transfusions, although this is rare [10].

\section{Pathophysiology}

Malaria infection develops via two phases: one that involves the liver (exoerythrocytic phase), and one that involves red blood cells or erythrocytes (erythrocytic phase). When an infected mosquito pierces a person's skin to take a blood meal, sporozoites in the mosquito's saliva enter the bloodstream and migrate to the liver where they infect hepatocytes, multiplying asexually and asymptomatically for a period of 8-30 days [11].

After a potential dormant period in the liver, these organisms differentiate to yield thousands of merozoites, which, following rupture of their host cells, escape into the blood and infect red blood cells to begin the erythrocytic stage of the life cycle [11]. The parasite escapes from the liver undetected by wrapping itself in the cell membrane of the infected host liver cell [11].

Within the red blood cells, the parasites multiply further, again asexually, periodically breaking out of their host cells to invade fresh red blood cells. Several such amplification cycles occur. Thus, classical descriptions of waves of fever arise from simultaneous waves of merozoites escaping and infecting red blood cells [11].

The parasite is relatively protected from attack by the body's immune system because for most of its human life cycle it resides within the liver and blood cells and is relatively invisible to immune surveillance. However, circulating infected blood cells are destroyed in the spleen. To avoid this fate, the P. falciparum parasite displays adhesive proteins on the surface of the infected blood cells, causing the blood cells to stick to the walls of small blood vessels, thereby sequestering the parasite from passage through the general circulation and the spleen [12]. The blockage of the microvasculature causes symptoms such as in placental malaria [13]. Sequestered red blood cells can breach the blood-brain barrier and cause cerebral malaria [12].

\section{Materials and Methods}

\section{Materials}

Human subjects were used Questionnaires

\section{Questionnaire Design}

Contents of the questionnaire include;

a) Frequency of malaria symptoms appearance

b) Use of malaria Prophylaxis

c) Malaria symptoms usually noticed

d) Type of exercise and frequency of exercise

e) Reason for Exercise

\section{Methodology}

Study location and reason for location: The location of this study was Bingham University, Karu, Nasarawa state, Nigeria. This location was selected because it presented the best and easiest means of getting regularly exercising individuals and sedentary individuals. It is also marshy thereby providing a good atmosphere for mosquito breed.

\section{Study Design}

The study was carried out to determine the frequency of malaria occurrence in regularly exercising subjects compared to non-exercising subjects within a period of three months with regular checks every two weeks on each of the subjects. The subjects were then administered questionnaire at the end of the twelve weeks. The subjects were divided as into four groups as follows;

GROUP A; Comprised of Non-exercising females GROUP B; Comprised of regularly exercising females (At least 3 times a week)

GROUP C; Comprised of Non-exercising males

GROUP D; Comprised of regularly exercising males (At least 3 times a week)

\section{Sampling Methods}

The healthy human subjects were selected from Bingham University community. The selection was carried out to include an equal number of males and females i.e. 30 males and 30 females, making a total of 60 . Out of this number, the subjects were divided into four groups (group A to D) each group comprising of 15 subjects. 
The subjects selected for this study were all healthy and free from malaria infection as at the time of selection

\section{Sampling Method}

Systematic random sampling method was used in selecting the subjects.

\section{Monitoring of Subjects}

The subjects were monitored for a total period of 12 weeks with their malaria status being checked every week. After this period, the subjects were given questionnaire to fill.

\section{Statistical Analysis}

In this project work, the following statistical parameters were used in analyzing the results

Mean $=\Sigma \mathrm{x} / \mathrm{N}$

Standard deviation

One way ANOVA

\section{Results}

\begin{tabular}{|c|c|c|c|c|c|}
\hline \multirow{4}{*}{ Frequency of exercise } & & $\mathbf{N}$ & Mean & Standard Deviation & Standard Error \\
\cline { 2 - 6 } & 1 & 15 & 0 & 0 & 0 \\
\cline { 2 - 6 } & 2 & 15 & 2.4 & 0.82808 & 0.21381 \\
\cline { 2 - 6 } & 3 & 15 & 0 & 0 & 0 \\
\cline { 2 - 6 } & 4 & 15 & 4.0667 & 1.90738 & 0.49248 \\
\hline \multirow{5}{*}{ Duration } & Total & $\mathbf{6 0}$ & $\mathbf{1 . 6 1 6 7}$ & $\mathbf{2 . 0 0 9 2 3}$ & 0.25939 \\
\hline \multirow{5}{*}{ Malaria } & 1 & 15 & 0 & 0 & 4.65986 \\
\cline { 2 - 6 } & 2 & 15 & 46 & 18.04756 & 0 \\
\cline { 2 - 6 } & 3 & 15 & 0 & 0 & $\mathbf{3 . 5 6 1 1 4}$ \\
\cline { 2 - 6 } & 4 & 15 & 52.6667 & 15.79632 & 0.243 \\
\hline & Total & $\mathbf{6 0}$ & $\mathbf{2 4 . 6 6 6 7}$ & $\mathbf{2 7 . 5 8 4 4 9}$ & 0.13093 \\
\cline { 2 - 6 } & 1 & 15 & 1.2 & 0.94112 & 0.20625 \\
\cline { 2 - 6 } & 2 & 15 & 0.4 & 0.50709 & 0.12599 \\
\cline { 2 - 6 } & 3 & 15 & 0.7333 & 0.79881 & $\mathbf{0 . 0 9 9 9 1}$ \\
\hline
\end{tabular}

Table 1: Mean and Standard Deviation.

\begin{tabular}{|c|c|c|c|c|c|c|}
\hline & & $\begin{array}{c}\text { Sum of } \\
\text { Squares }\end{array}$ & $\begin{array}{c}\text { Difference of } \\
\text { square }\end{array}$ & $\begin{array}{c}\text { Mean of } \\
\text { Square }\end{array}$ & Frequency & Significance \\
\cline { 2 - 6 } & Between Groups & 177.65 & 3 & 59.217 & 54.782 & 0 \\
Frequency & Within Groups & 60.533 & 56 & 1.081 & & \\
of Exercise & Total & 238.183 & 59 & & & \\
Duration & Between Groups & 36840 & 3 & 12280 & 85.391 & 0 \\
Malaria & Within Groups & 8053.333 & 56 & 143.81 & & 0.006 \\
& Total & 44893.333 & 59 & & & \\
& Between Groups & 7.067 & 3 & 2.356 & 4.667 & \\
& Within Groups & 28.267 & 56 & 0.505 & & \\
& Total & 35.333 & 59 & & 54.782 & \\
\hline
\end{tabular}

Table 2: ANOVA. 


\section{Discussion and Conclusion}

\section{Discussion}

From the study carried out to relate the frequent exercise to malaria occurrence, the results showed a negative correlation between frequent exercise and malaria occurrence. After a study period of twelve weeks, there was a significant difference in malaria attack occurrences in the two groups with the non-exercising group having a more significant mean of malaria occurrence than the regularly exercising group in both the male subjects and the female subjects (non-exercising female group mean: (1.2), nonexercising male group mean: (0.7)) compared to the regularly exercising group (mean of regularly exercising female group: (0.4), regularly exercising male subjects mean: $(0.3)$ ).

The regularly exercising male group recorded a higher overall duration of exercise per day compared to the female regularly exercising students (male regularly exercising group mean per day: (53 minutes), female regularly exercising group mean per day: (46 minutes per day)).

Exercise has been reported to cause change in antibodies and white blood cells. White Blood Cells are the body's immune system cells that fight disease. These antibodies or White Blood Cell circulate more rapidly during exercise. So they could detect illnesses faster they will in the absence of exercise. Also, the brief rise in temperature during and right after exercise may prevent micro-organisms from growing thereby helping the body to fight infections. Exercise is also known to slow down release of some stress hormones thereby protecting against infections [14].

Malaria occurrence was only slightly affected by gender as there was only a slight difference between the male group and the female group in occurrence of malaria in both the regularly exercising subjects and the non-exercising subjects; this was expected as the male subjects are generally more active than their female counter-parts.

These results prove a need to further explore the benefits of exercise as a preventive measure in communicable diseases by carrying out further longitudinal studies in larger population samples and larger population variables to further ascertain the importance of exercise in reducing the frequency of occurrence of communicable diseases especially.

Exercise in this study deviated from the routine research on importance of exercise in non-communicable diseases to show that exercise can also have positive effects on the communicable diseases.

\section{Conclusion}

The study has shown an important correlation between malaria and exercise; a regular exercise can impact the frequency of occurrence of malaria in regularly exercising individuals. Regularly exercising individuals report less malaria cases over time compared to non-exercising individuals.

This study further broadens knowledge on the importance of exercise in improving the health and wellbeing of individuals. It also emphasizes the benefits of exercise and the need to try to engage in exercise as much as possible.

\section{Recommendation}

Further research should be done to find how exercise affects other diseases, especially the communicable diseases.

\section{References}

1. Wilmore J, Knuttgen H (2003) Aerobic Exercise and Endurance Improving Fitness for Health Benefits. The Physician and Sportsmedicine 31(5): 45.

2. Webb JLA (2009) Humanity's Burden: A Global History of Malaria. Cambridge University Press.

3. Tan SY, Sung H (2008) Carlos Juan Finlay Of mosquitoes and yellow fever. Singapore Medical Journal 49(5): 370371 ncbi.nlm.nih.gov/pubmed/18465043

4. Caraballo H (2014) Emergency department management of mosquito-borne illness. Emergency Medicine Practice, 16(5).

5. Taylor WR, Hanson J, Turner GD, White NJ, Dondorp AM, et al. (2011) Redefining cerebral malaria by including malaria retinopathy. Future Microbiology, 6(3): P.34955. doi:10.2217/fmb.11.3.

6. Bartoloni A, Zammarchi L (2012) Clinical aspects of uncomplicated and severe malaria. Mediterranean Journal of Hematology and Infectious Diseases 4(1): e2012026. doi:10.4084/MJHID. 026.

7. Beare NA, Lewallen S, Taylor TE, Molyneux ME (2011) Redefining cerebral malaria by including malaria retinopathy. Future Microbiology 6(3): 349-355.

8. Cowman AF, Berry D, Baum J (2012) The cellular and molecular basis for malaria parasite invasion of the human red blood cell. Journal of Cell Biology 198(6): P.961-71. 
9. Arrow KJ, Panosian C, Gelband H (2004) Institute of Medicine (US). Committee on the Economics of Antimalarial Drugs, Saving Lives, Buying Time: Economics of Malaria Drugs in an Age of Resistance. National Academies Pres, P. 141.

10. Owusu-Ofori AK, Parry C, Bates I (2010) A review of the literature from sub-Saharan Africa. Clinical Infectious Diseases 51(10): 1192-1198.

11. Bledsoe GH (2006) Malaria primer for clinicians in the United States. Southern Medical Journal 98 (12):
$1197-1204$

12. Tilley L, Dixon MW, Kirk K (2011) The Plasmodium falciparum-infected red blood cell. International Journal of Biochemistry and Cell Biology 43(6): 839-842.

13. Mens PF, Bojtor EC, Schallig HD (2012) Molecular interactions in the placenta during malaria infection. European Journal of Obstetrics \& Gynecology and Reproductive Biology 152(2): 126-132.

14. Exercise and Immunity, MedlinePlus, US National Library of Medicine, Feb, 2020. 